\title{
LA NARRATIVA DE ELENA FERRANTE: EL DESEQUILIBRIO DE OLGA, UNA MEDEA MODERNA ${ }^{1}$
}

\author{
María Reyes Ferrer ${ }^{2}$
}

\begin{abstract}
La narrativa de Elena Ferrante: El desequilibrio de Olga, una Medea moderna
Resumen: El presente trabajo pretende acercarse a la figura de la enigmática escritora Elena Ferrante y analizar algunas de las características más destacables de su narrativa. Para ello, nos centraremos en una obra en concreto, I giorni dell'abbandono, y las vivencias de Olga, su protagonista, que guiará al lector por los entresijos de su agonía personal tras ser abandonada y traicionada por su marido.
\end{abstract}

Palaras clave: Elena Ferrante, Olga, abandono, maternidad, existencia.

\section{Elena Ferrante's Narrative: Olga's Instability, a Modern Medea}

Abstract: The present work aims to approach the image of the enigmatic writer Elena Ferrante and analyze some of the most distinctive features of her narrative. To do so, we will focus on a particular work Il giorni dell 'abbandono and Olga's -its protagonist- experiences, which will guide the reader through the in and outs of her own agony after being dumped and betrayed by her husband.

Key words: Elena Ferrante, Olga, abandonment, maternity, existence.

\section{Introducción}

La escritora italiana Elena Ferrante ha revolucionado el panorama literario contemporáneo desde una doble vertiente: por un lado, sorprende con una ficción realista centrada en distintos aspectos del universo femenino y, por otro, desconcierta con su identidad, desconocida hasta la fecha, y se esconde tras un falso apelativo. Tomando como punto de partida este último argumento, el éxito literario de la escritora italiana, o quizás escritor ${ }^{3}$, radica en buena parte en el misterio que se ha creado alrededor de su persona, principalmente debido a su anonimato y al distanciamiento voluntario de los

${ }^{1}$ Fecha de recepción: 04/11/2015.

Fecha de aceptación: 21/12/2015.

${ }^{2}$ Profesora Asociada, Departamento de Filología Francesa, Italiana, Románica y Árabe, Área de Filología Italiana, Universidad de Murcia; $\square$ maria.reyes1@um.es.

${ }^{3}$ Las especulaciones acerca del sexo de Elena Ferrante, sobre todo a través de la prensa italiana, han desatado ciertas polémicas y son muchas las estudiosas que, tras analizar su escritura y acercarse al texto, rechazan unánimemente que Ferrante sea en realidad un hombre: “it's not that male writers haven't created dynamic women since time began, but rare is such exquisite attention paid to a woman's relationship with other women, or their children who both connect and distract them from the world" (Valby 2014). 
círculos mediáticos y literarios ${ }^{4}$. Ferrante trata de huir de la corriente mediática y del proceso de personalización de las obras a través del cual escritores y libros se convierten en mercancía que vende. La escritora reconoce no querer entrar en el juego editorial y evita establecer cualquier tipo de relación entre el libro y el autor ya que considera los dos elementos autónomos, distintos y de los que mantenerse distante. Se cuestiona hasta qué punto es importante conocer al escritor una vez leída la obra -"io penso che la buona novella sia sempre: è uscito un libro che vale la pena di leggere" (Ferrante 2003: 20)- y prefiere tener la libertad de escribir sin ser reconocida, desde el espacio privado e íntimo que le permite afrontar la escritura sin normas ni convenciones. Cuanto más se aleja de su obra y más la priva de una identidad o de un cuerpo, más autonomía tiene el texto para convertirse en lo que pretende ser, una "invenzione romanzesca", y la cercanía a él sólo implicaría llenar el texto de realidad, de ella misma, perdiendo su condición de "romanzo": "Voglio, perciò, che il mio romanzo se ne vada il più lontano possibile proprio possa dare la sua verità romanzesca e non gli scampoli accidentali, che pure contiene, di autobiografia" (Ferrante 2003: 63). Elena Ferrante replantea desde otra perspectiva y desde otro tiempo una preocupación similar a la ya planteada por Alessandro Manzoni acerca de los efectos que puede tener la presencia del autor en el texto y la contaminación de la obra con su creador.

Siente, por lo tanto, la necesidad de liberarse de su creación una vez que el estricto trabajo del escritor ha finalizado, y considera oportuno dejarla ir hacía a un público que tendrá total libertad de interpretación del texto, emitiendo juicios independientes sobre la historia y su creadora. La negación del autor se refleja también en las portadas de sus libros donde aparecen mujeres sin rostros o con rostros parcialmente velados.

\footnotetext{
${ }^{4}$ Este es un aspecto que ha llamado la atención notablemente e incluso ha despertado el interés fuera de las fronteras italianas. Basta leer La Frantumaglia (2003, Roma: E/O) -una obra que recoge un considerable número de entrevistas que ha realizado por escrito-, para observar como una de las preguntas que más se repite es el porqué de su anonimato y la insistencia de permanecer en la sombra. Sin embargo, este aspecto no siempre ha sido bien acogido y la escritora ha recibido duras críticas, especialmente la de Sebastiano Vassalli con motivo de su nominación al Premio Strega: "Ci augureremo che vinca, come al solito, un peggiore: un essere umano" (Vassalli 2015: 33).

${ }^{5}$ Ferrante, en su carta de respuesta a Francesco Erbani se cuestiona unas cuantas preguntas "retóricas" acerca de lo verdaderamente importante para un libro, como el hecho de conocer al escritor o, como ella misma define, al personaje del escritor: 'la risonanza dell'autore, o per dire meglio del personaggio d'autore che va in scena grazie ai media, è un supporto fondamentale per il libro?” (Ferrante 2003: 23).
} 
No obstante, la crítica literaria Laura Buffoni ve ciertas contradicciones entre la intención de mantener su identidad velada y las posibles referencias autobiográficas que se encuentran en el texto literario. Concretamente Buffoni menciona la ciudad de Nápoles, "descritta come solo chi ci è cresciuto potrebbe fare” (Buffoni 2014), y la voz narrativa que va asumiendo rasgos más concretos hasta llegar a lo que denomina un "Io da autofiction". Es probable que la autora, en su necesidad de escritura, deje pequeñas huellas de sí misma en el texto y su anonimato sea la forma de protegerse de su propia creación ${ }^{6}$, "di proteggermi dai suoi effetti", como afirma. Ferrante ha declarado en numerosas ocasiones su cercanía con el texto, con los personajes que crea y el mundo que recrea. Es más, paradójicamente, la escritora no esconde que la trama de sus narrativas nace de las experiencias vividas, de recuerdos, de gente conocida, que le servirán de empuje para dar rienda suelta a su creatividad:

[...] real events don't count much when one writes; at most they are like getting shoved while out on the street. Rather, a story is a deep chasm of very different experiences that have accumulated over the course of a lifetime, and that miraculously nourish events and characters in the story. There are some experiences that are difficult to use, that are elusive, embarrassing, at times unsayable, because they belong to us so intimately. I am in favor of stories that are fed by these kinds of experience. (Ferrante en Valby 2014)

El hecho de publicar un libro supone, para la escritora, un acto que implica romper con el anonimato al ceder partes íntimas de uno mismo: "decidere di offrire ad altri, nella forma che ci sembra la più adeguata, ciò che intimamente ci appartiene" (Ferrante 2003: 26). Publicar puede significar mostrar una faceta propia e íntima, pero también se destaca la alteridad que sufre el escritor cuando se encuentra en pleno proceso creativo, aunque hasta la fecha no se conocen sus influencias o inspiraciones literarias": "I vecchi miti

\footnotetext{
${ }^{6}$ El hecho de ocultar su identidad no sirve únicamente para no entrar en el juego mediático o protegerse del texto sino que el ser anónima le reporta, en cierto sentido, un beneficio creativo: "Chiudo con questo tema dicendo per ultimo che scrivere sapendo di non dover apparire genera uno spazio di libertà creativa assoluta. $\grave{E}$ un angolo mio che intendo difendere, ora che l'ho sperimentato. Se ne fossi privata, mi sentirei bruscamente impoverita" (Ferrante 2003: 63).

${ }^{7}$ A pesar de advertir influencias de otros durante el proceso creativo, Ferrante huye de filiaciones literarias concretas, con Elsa Morante o con Anna Maria Ortese, aunque admite ser ávida lectora de ambas.
} 
sull'ispirazione forse dicevano almeno una verità: quando si fa un lavoro creativo si è abitati da altro, in qualche misura si diventa altro. Ma quando si smette di scrivere si ridiventa se stessi, la persona che comunemente si è, nelle occupazioni, nei pensieri, nel linguaggio" (Ferrante 2003: 38). Ferrante hace una neta distinción entre el momento en el que se convierte escritora y se deja habitar por otros, y cuando vuelve a su mundo real, a una rutina común. La parte de escritora parece fragmentarse en diversos pensamientos y se llena de conceptos que giran en torno al artificio literario, algo que no tiene cabida en su cotidianidad. Es precisamente la cotidianidad de la que se sirve para crear y recrear sus historias y sus personajes, enriqueciendo su narrativa con estilo muy personal y directo.

\section{Aspectos relevantes de la narrativa de Elena Ferrante}

Elena Ferrante explora, a través de la escritura, todos los aspectos de la experiencia femenina -maternidad, amistad, femineidad, sexualidad- que conducirán a una serie de reflexiones existenciales formuladas a través de los pensamientos de los personajes, quienes tratan de ahondar en sus problemas y reconducir sus vidas. La existencia de estas mujeres está ligada a un profundo malestar, al inconformismo y a la inadecuación social. Son mujeres que sufren fuertes desequilibrios emocionales y comienzan una ardua andadura hacia su reconstrucción personal, cuestionando los roles femeninos y los diferentes vínculos que se crean entre las mujeres. Respecto a este punto, la escritora propone diferentes dimensiones en lo que respecta a las posibles relaciones que se establecen entre mujeres: madre-hija, amigas, mujer-mujer, mujer-espejo.

Su narrativa está centrada en el análisis psicológico y en la construcción o búsqueda de la identidad de las protagonistas que, tras verse envueltas en cruciales circunstancias psicológicas y emotivas, se ven obligadas a dar un giro brusco de personalidad o buscar reminiscencias de un pasado que les guíen hacía una posición estable en el presente. Para ello, Ferrante hace un profundo estudio del interior de las mujeres, "[le] loro inquiete e 
sconcertanti vite interiori -fatte di sentimenti esasperati, pensieri confusi, ansie e dubbi" (Rooy 2007: 159) con una notable carga emotiva.

Las vivencias que experimentan sus personajes están contextualizadas en un doble espacio: uno más superficial y lleno de lugares comunes para el lector, como son los ambientes de clase media y las ciudades conocidas como Nápoles, que adquiere un significado complejo en la obra, y un contexto más profundo y trascendental que tiene lugar en el espacio doméstico donde se "rasga la piel de lo habitual" (Wood 2013) y deja entrever las entrañas de las mujeres, sus pensamientos y sus sensaciones, sin artificio alguno.

El impacto que ocasiona la lectura se debe, en buena parte, al estilo narrativo que la escritora cultiva en cada una de sus novelas. A pesar de utilizar una prosa relativamente sencilla, directa y escasa en descripciones físicas, Badami (2015) señala que en su narrativa abundan los elementos viscerales, sensuales o táctiles que sirven para expresar sentimientos que, en ocasiones, son difíciles de admitir, y se materializan físicamente y a través de la fusión entre lo interno y lo externo. Existe un continuo movimiento entre los hechos externos que acontecen y el fluir de los pensamientos y emociones de las protagonistas, utilizando para ello la técnica del stream of consciousness que permitirá la construcción de la realidad desde una doble óptica, pública y privada. A continuación, pasaremos a analizar estos elementos en I giorni dell'abbandono, una novela con una fuerte carga emocional que narra la agonía de su protagonista tras ser abandonada por su marido.

\section{I giorni dell'abbandono: la agonía de Olga}

Desde el inicio de la novela, ya en las primeras líneas, es posible advertir la urgencia que siente Olga, la protagonista, por narrar su historia: "Un pomeriggio d'aprile, subito dopo pranzo, mio marito mi annunciò che volveva lasciarmi. Lo fece mentre sparecchiavamo la tavola, i bambini litigavano come al solito nell'altra stanza, il cane 
sognava brontolando accanto al termosifone" (Ferrante 2002: 7). Estas líneas, entre las que se puede leer una crueldad implícita por el fuerte contraste que se establece entre la noticia y la escena familiar, dan paso a I giorni dell'abbandono, la historia de una mujer de 38 años, casada y con dos hijos, Ilaria y Gianni, cuya vida familiar acaba de romperse $y$, junto a ella, su propia vida. Olga narra en primera persona el sufrimiento causado por el abandono y la traición de Mario, su marido, y cómo la ausencia de este en el hogar desfragmentará su identidad, y se verá forzada a arrastrar como un peso insoportable su condición de mujer, la maternidad, la soledad y su propia existencia.

A pesar de lo que pueda parecer a simple vista, y como Martine Bovo-Romoeuf (2006) sostiene, la trama de la novela no trata tanto de una historia basada en la experiencia del abandono sino de una situación de desgaste sentimental y emocional que le provocará una fuerte sacudida de sus convicciones, de su forma de ser, y todo ello repercutirá en el agotamiento de su cuerpo y de su mente. La propia Ferrante afirma que la novela se escribió con el objetivo de profundizar en lo que denomina "una storia di destrutturazione" (Ferrante 2003: 61), y no en el fatídico hecho conyugal. La escritora considera que una de las consecuencias del desamor es la fuerte desestabilización de los parámetros culturales por los que se trabaja a lo largo de la vida y las consecuencias que esto tiene en las personas:

Gli esseri umani danno il peggio di sé quando i loro abiti culturali si lacerano ed essi si trovano di fronte alla nudità dei loro organismi, ne sentono la vergogna. In un certo senso la sottrazione dell'amore è l'esperienza comune più vicina al mito della cacciata dal paradiso terrestre, è la fine violenta dell'illusione di avere un corpo celeste, è la scoperta della propria inessenzialità e deperibilità. (Ferrante 2003: 61-62)

Utilizando una técnica introspectiva, Olga se va desnudando poco a poco frente al lector. Comienza su monólogo narrativo con un notable sosiego ante la inesperada noticia, una actitud que se distancia de la rabia, la turbación y la incomprensión que traerán los 
días venideros ${ }^{8}$. Observando sus cambios, es posible individualizar tres períodos por los que atraviesa la protagonista tras la marcha de Mario: uno de confusión e incertidumbre, otro de ira y abandono físico y personal y, finalmente, un último de reconstrucción personal y mayor control emocional.

\subsection{Olga, una Medea moderna}

Olga es una Medea moderna que trata de reaccionar contra el abandono, es una Monique $^{9}$ de Beauvoir, aunque con mayores posibilidades de éxito al huir de la victimización, como Stefania Lucamante señala: "Ferrante empathizes with her, avoiding victimization and condemnation and pragmatically looking for a position in society for her” (2008: 85). Olga rozará la locura pero su voluntad por distanciarse del personajefantasma la "poverella" y de las mujeres de La femme rompue, a quienes califica como "donne stupide" que se dejan romper por hombres, le salvará de caer definitivamente en el abismo. Las reiteradas preguntas acerca de su identidad y los soliloquios que mantiene con ella misma para calmarse, tratar de razonar, evitar descuidos o realizar pequeñas rutinas a fin de no perder la cabeza ayudarán a la protagonista a salir de su agonía personal. A simple vista, y tras la noticia de Mario, Olga se mostrará en actitud controlada y en aparente calma en un primer momento. Sin embargo, en una lectura más profunda, su muestra de serenidad está estrechamente relacionada con una fuerte represión como consecuencia, en parte, de la educación recibida y de su entorno natal, factores que obstaculizarán la libre expresión de los sentimientos, mermados por los límites expresivos que se ha autoimpuesto para evitar dar una imagen alterada de sí misma: "Anche per tenere sotto controllo l'angoscia dei mutamenti mi ero definitivamente abituata ad

\footnotetext{
${ }^{8}$ En las novelas de Elena Ferrante es recurrente la pérdida del autocontrol por parte del narradorprotagonista y en el estilo del texto, ambos alejándose de la aparente calma inicial, como Elizabeth Alsop propone en su análisis de I giorni dell'abbandono y L'amore molesto: "In fact, both novels betray a marked fascination with the theme of collapse - with the moments in which such systems of surveillance falter, or even fail. At the same time, however, they consistently underscore the importance of such surveillance in the struggle for survival" (2014: 466-467)

${ }^{9}$ Cfr. Beauvoir 1967.
} 
aspettare con pazienza che ogni emozione implodesse e prendesse la via della voce pacata, custodita in gola per non dare spettacolo di me" (Ferrante 2002: 11). Con la palabra "mutamenti", Olga se refiere a los continuos cambios de vida por los que atravesaba la pareja debido al trabajo de Mario que, con frecuencia, era destinado a diversos lugares que implicaban un continuo cambio de rutina. Olga deja su trabajo y su vida, dedicándose por completo a seguir a su marido en su andadura profesional, algo que tras su marcha lamentará

A esta disciplina autoimpuesta Ferrante la llama "la sorveglianza", una vigilancia psicológica que controla los sentimientos y la aleja de su esencia personal, de su infancia napolitana que pretende olvidar mostrando un comportamiento poco impulsivo y complaciente. Olga alude a su infancia y al núcleo familiar, y la recuerda como un período caracterizado por "sentimenti rumorosi, esibiti" (10), con gestos alborotados y reacciones incontroladas, algo que pretenderá evitar autodisciplinándose en un control excesivo de sus formas y los tonos de voz.

Además, el control no sólo se ejerce en el plano psicológico sino también corporal, tratando de gobernar cada movimiento, especialmente en su segunda fase del abandono, donde su cuerpo se presenta fuera de control. En estos primeros días de calma forzada, Olga comienza a analizar su condición de "prisionera" del hogar en la que se había convertido durante sus años de matrimonio, en parte inducida por el marido y en parte por voluntad propia, cediendo cada una de sus ambiciones, entre ellas la escritura, al cuidado del hogar y de la familia. Hasta la fecha, Olga no se había planteado la dirección que su vida había tomado, si era un deseo personal, y únicamente se dedicaba a cubrir las necesidades familiares. No obstante, la sensación de angustia comienza a crecer cuando la protagonista concluye que, sin Mario, la dedicación al hogar ya no es una elección sino es una obligación: "I bambini, il cane, la spesa, il pranzo e la cena, il danaro. Tutto mi segnalava le conseguenze pratiche dell'abbandono" (20).

La resignación de Olga a su condición de madre y esposa es inmediata. La protagonista asume que, tras el abandono, las responsabilidades del hogar están implícitas 
en su condición de mujer, y no se cuestiona una posible división de tareas con Mario ${ }^{10}$, quien desaparece dejando todo atrás y anulando cualquier tiempo y espacio de reflexión para Olga, sin la intromisión de los hijos o las múltiples ocupaciones a las que se ve obligada atender. El hecho de ser abandonada, por lo tanto, le exige arrastrar el peso de la rutina y del hogar, que parece convertirse en el núcleo fundamental de su condición de mujer.

El análisis de sus años de matrimonio y la lenta aceptación del peso que supone el abandono le traerán a la mente una imagen de su infancia napolitana, la de una mujer abandonada conocida como la "poverella": "La donna perse tutto, anche il nome (forse si chiamava Emilia), diventò per tutti "la poverella", cominciammo a parlarne chiamandola così" (15). La figura de la "poverella" estará presente a lo largo de toda la novela como una sombra negra que planea sobre Olga, un fantasma de un pasado que marcó la infancia de la protagonista hasta tal punto que parte de su represión y control sentimental es consecuencia de su traumático recuerdo, como Lucamante (2008) señala.

A partir de este momento comienza la segunda fase de transición en la vida de Olga, la más larga y la más áspera, en la que trata de reaccionar constantemente contra el malestar del abandono, huyendo de la imagen de la "poverella", siempre envuelta en lágrimas, y de la propia Monique, por su falta de control y debilidad. En este período, la vida de Olga está marcada por la ira y el abandono, como se apuntó anteriormente.

\subsection{La ira de Olga: reflexiones sobre la maternidad.}

Respecto a la ira, la escritora logra transmitir la violencia del sentimiento especialmente a través del lenguaje:

\footnotetext{
${ }^{10}$ Elena Ferrante justifica en cierta medida la actitud de Mario, de quien dice: "è solo un uomo che ha smesso di amare la donna con cui vive e si scontra con l'impossibilità di spezzare quel legame senza umiliarla, senza farle del male. I suoi comportamenti sono quelli di un essere umano che priva un altro essere umano del suo amore" (Ferrante 2003: 69). Sin embargo, a pesar de ser una situación fuera de control por pertenecer al campo de los sentimientos, Ferrante no hace referencia a la carga que supone el abandono para Olga, al espacio que Mario se permite tomarse, sin hijos y sin la carga del hogar, mientras la protagonista se ahoga en un sufrimiento que está obligada a compaginar con las obligaciones maternales.
} 
Cominciai a cambiare. Nel giro di un mese persi l'abitudine di truccarmi con cura, passai da un linguaggio elegante, attento a non urtare il prossimo, a un modo di esprimermi sempre sarcástico, interrotto da risate un po' sguaiate. Piano piano, malgrado la mia resistenza, cedetti anche al linguaggio osceno. L'oscenità mi veniva alla labbra con naturalezza, mi pareva che servisse a comunicare ai pochi conoscenti che ancora cercavano frígidamente di consolarmi che non ero una che si fa abbindolare con le belle parole. Appena aprivo la bocca sentivo la voglia di irriddere, macchiare, insozzare Mario e la sua troia. (Ferrante 2002:27)

Poco a poco Olga, en su decadencia, se acerca a la figura de la "poverella", descuidada, deslenguada y triste. Comienza a utilizar un lenguaje ordinario y abrupto en el trato con las personas no solo como un sistema de protección ante las adversidades sino también como una herramienta de empoderamiento al sentirse capaz de superar los límites de las normas de socialización y romper con las barreras de la auto-contención, dos hechos que apuntan a su entrada en un abismo de que será difícil salir.

Especialmente remarcable es el uso del lenguaje obsceno - un registro por el que Olga confiesa haberse sentido atraída desde pequeña por identificarse con una libertad masculina-, sobre todo cuando imagina a Mario y a su amante, Carla, manteniendo relaciones. Los pensamientos y los soliloquios de Olga, detallados minuciosamente con un lenguaje erótico, obsceno y gráfico, muy directo, son sin duda uno de los aspectos narrativos más logrados de la novela al aportar una gran intensidad y ser el elemento más fiable para identificar los cambios que sufre Olga, aportando además veracidad a la historia. El uso del lenguaje obsceno, las palabras malsonantes, van sucedidas también de imágenes de repulsa hacia su propio cuerpo. Imagina el cuerpo joven de Carla, su delicada piel, sus senos firmes y, en definitiva, un cuerpo que todavía no ha experimentado la maternidad, y lo compara con su propia situación. Olga comienza a concebir la maternidad no solo como una carga diaria de un sinfín de tareas para atender a los hijos, sino como la causa del desgaste del cuerpo de la madre. La madre cede de su tiempo y también de su propio espacio, un espacio corporal que se sacrifica para dar vida a los hijos. Cuando alude explícitamente a la maternidad, la figura de Olga y la "poverella" se acercan creándose un paralelismo entre ellas: las dos emanan un "malodore di mamma" 
(101). Olga ahonda en las raíces profundas de la maternidad y le produce repulsión el hecho de amamantar a los hijos como lo hacen los animales o manchar su cuerpo con grumos o vómitos. A través de la maternidad, se pone de manifiesto una de las mayores contradicciones entre las que se mueve la vida de Olga: al inicio de la novela, la protagonista se presenta como una madre entregada a su obligación, que son sus hijos y, sin embargo, se observa un fuerte desequilibrio entre la imagen social proyectada y sus reflexiones existenciales, un hecho que se exteriorizará cuando pierde el interés por la proyección social tras el abandono de Mario.

La fase inicial de su maternidad es traumática y prueba de ello es el testimonio que deja a través de la escritura. Resulta interesante analizar la función que tiene la escritura para Olga, quien pone por escrito pensamientos y reflexiones que jamás verbalizaría por un exceso de autocontrol como se comentó con anterioridad:

Scrivevo anche, a tratti, tra le cifre, come mi sentivo: un grumo di cibo che i miei figli masticavano in continuazione; un bolo fatto di materia viva che amalgamava e ammorbidiva continuamente la sua sostanza vivente per permettere a due sanguisughe voraci di nutrirsi lasciandomi addosso l'odore e il sapore dei loro succhi gastrici [...] Per quanto mi lavassi, quel malodore di mamma non se ne andava. (101)

Para Olga, existe un fuerte desencuentro entre la maternidad y la feminidad; los hijos destruyen el cuerpo de la madre y aniquilan al sujeto privándole de lo femenino y, por tanto, de sí misma, convirtiendo su cuerpo en el "corpo di un incesto" (102). Lejos de asimilar la maternidad como un proceso creador de vida, la maternidad se convierte para nuestra protagonista en dolor corporal, humillación y la muerte del sujeto femenino. Olga se define como "la madre da violare, non un'amante" (102), y llega a empatizar con Mario, a quien achaca la búsqueda de un cuerpo joven como el de Carla, libre de cargas y con quien explorar fantasías, para poder librarse de la culpabilidad de corromper sexualmente un cuerpo ya corrompido por los hijos, un cuerpo no femenino. 
El concepto que Olga tiene de su cuerpo, de la maternidad, está íntimamente ligado al concepto kristevano de lo abyecto, que se define como: "algo rechazado del que uno no se separa, del que uno no se protege de la misma manera que de un objeto. Extrañeza imaginaria y amenaza real, nos llama y termina por sumergirnos" (Kristeva 1989: 11). Kristeva sostiene que lo que vuelve abyecto al cuerpo son los elementos que trastornan "una identidad, un sistema, un orden" (Íbidem). Olga experimenta la abyección física, tangible, a través de los residuos de comida que sus hijos le dejan sobre el cuerpo, y en un plano psicoanalítico, basada en la "pérdida inaugural fundante de su propio ser" (Kristeva 1989: 12). Obsesionada con la imagen sexual de Mario y Carla, y su rechazo/desconcierto sobre ella misma, Olga decide seducir a Carrano, su misterioso vecino, para reconocerse a sí misma a través del deseo de éste, del deseo del otro en términos lacantianos. La mujer busca ser reconocida por Carrano para afirmarse en el deseo ajeno y alejarse de cualquier forma de posesión del cuerpo, buscando "el reconocimiento de la propia subjetividad" (Zadra 2006). Olga no siente ningún tipo de atracción por su vecino y, sin embargo, está dispuesta a mantener relaciones con él para volver a sentirse deseada, para confirmar que aún puede ser una mujer -(madre)atractiva: "Dissi aspetta, lo respinsi, mi sollevai, mi tolsi la camicetta, mi slacciai il reggiseno. Chiesi stupidamente: ti piacciono, era un'ansia che mi stava crescendo, volevo che mi ripetesse il suo consenso" (Ferrante 2002: 90). Para sentirse deseada, Olga está dispuesta a ceder, a su vez, a todos los deseos de Carrano, entregándole su cuerpo y materializando cualquier fantasía que este tenga para conseguir su objetivo: "dappertutto ti devo piacere" (94). Según Lacan, el acto sexual está más relacionado con la construcción de fantasías, con el ego, que con el plano material, el cuerpo, un aspecto que puede llegar a suscitar repugnancia. Olga construye una fantasía en torno a Carrano para sentirse deseada, aunque después sentirá rechazo hacía él al volver a tomar conciencia sobre su propio cuerpo. La "poverella" vuelve a entrar en escena y, tras abandonar la casa de Carrano, oye la voz de la mujer napolitana que con actitud desaprobatoria dice: "Io sono pulita sono vera gioco a carte scoperte" (96). La aparición vuelve a recrear la abyección de Olga hacia el cuerpo sucio, deshabitado y abatido por la situación. 
El día después de su encuentro con Carrano será el día más agónico tras el abandono, pero será el día en el que inicie su recuperación. Olga se verá obligada a hacer frente a dos situaciones complicadas: la enfermedad de su hijo y la muerte del perro, Otto, que logrará superar y que le ayudarán a recomponer los fragmentos de sí misma: “[...] fossi solo un insieme sconnesso di lati, una foresta di gure cubiste ignota a me stessa [...]" (145). La construcción de Olga había sido modelada por Mario y su cuerpo había quedado habitado por lo que llama "un abbaglio di fine adolescenza, una mia illusione di stabilità". Detrás de la imagen de Olga existe una infinidad de mujeres abandonadas que pierden la cabeza. Como Lucamante (2008) argumenta, Olga arrastra una personalidad desconocida durante años, construyendo una existencia donde lo real y lo ficticio se yuxtaponen en escasa harmonía para beneficiar a otros.

\section{Conclusión}

En todas sus novelas, Elena Ferrante hace un extraordinario estudio de la mujer y del universo femenino, dos facetas que no dejan indiferente al lector por la inmediatez del texto y la veracidad que aporta a la historia a través del lenguaje, unas características necesarias para poder narrar a la mujer desde lo más profundo. En una narrativa compleja por la temática existencial, el personaje de Olga es tangible, posible en la vida real, y capaz de transmitir visiones de la vida y sentimientos cercanos al público que los lee. Olga sucumbe a las consecuencias del abandono y lo proyecta a través de la dicotomía del amor/odio materno, y del cuerpo maternal, factores que son más determinantes para la abyección que el abandono en sí mismo por parte de Mario. Atravesar momentos sórdidos y poco gratificantes como la relación sexual con Carrano o la convivencia con la omnimpresente $-\mathrm{y}$ casi obsesiva- imagen de la "poverella" son necesarios para la reconstrucción del "yo" tras la completa desfragmentación del mismo. La identificación total y parcial con la "poverella", como mujer y como madre, hará que Olga se cuestione su imagen y afronte sus temores, dos hechos que le permitirán iniciar una nueva etapa de 
su vida poniéndose como sujeto principal, y no como objetvo, de sus actuaciones y sus decisiones.

\section{Referencias bibliográficas}

Alsop, Elizabeth. 2014. "Femmes Fatales: “'La fascinazione di morte' in Elena Ferrante's L'amore molesto and I giorni dell'abbandono". Italica, vol 91, 3: 466- 485.

Badami, Sunil. 2015. "Who is She? And What Does It Matter?". Southerly Journal. http://southerlyjournal.com.au/2015/03/03/who-is-she-and-why-does-it-matter/. [9 sept. 2015].

Bovo-Romoeuf, Martine. 2006. "Sensualité et obscénité dans L'amore molesto et I giorni dell'abbandono d'Elena Ferrante". Images littéraires de la société contemporaine, 2: 129- 13. http://cei.revues.org/823?lang=fr. [20 sept. 2015].

Buffoni, Laura. 2014. "Elena Ferrante sono io". Internazionale. http://www.internazionale.it/opinione/laura-buffoni/2014/11/30/elena-ferrantesono-io. [3 oct. 2015].

De Beauvoir, Simone. 1967. La femme rompue. Paris: Gallimard.

Ferrante, Elena. 2002. I giorni dell'abbandono. Roma: Edizioni E/O. 2003. La Frantumaglia. Roma: Edizioni E/O.

Kristeva, Julia. 1989. Poderes de la perversión: ensayo sobre Louis-Ferdinand Céline, México: Siglo XXI.

Lucamante, Stefania. 2008. A Multitude of Women: The Challenges of the Contemporary Italian Novel. Toronto: University of Toronto Press.

Rooy, Ronald. 2007. "Identità, memoria e passato nell'opera di Patrizia Velduga e Elena Ferrante". La forma del passato. Questioni di identità in opere letterarie e cinematografiche italiane a partire dagli ultimi anni Ottanta. Eds. Sabina Gola y Laura Rorato. Bélgica: Peter Lang: 159-172.

Valby, Karen. 2014. "Who is Elena Ferrante? An interview with the mysterious Italian autor". Entertainment Weekly. http://www.ew.com/article/2014/09/05/elenaferrante-italian-author-interview. [3 oct. 2015]. 
Vassalli, Sebastiano. 2015. "Premio Strega: vinca il peggiore piuttosto che l'autore fantasma",

Il

Corriere

della

Sera:

33.

http://archiviostorico.corriere.it/2015/marzo/29/Premio_Strega_vinca_peggiore_pi uttosto_co_0_20150329_794059ce-d5da-11e4-b9e9-3309be854897.shtml. [24 sept. 2015].

Wood, James. 2013. "Women on the Verge". The New Yorker. http://www.newyorker.com/magazine/2013/01/21/women-on-the-verge. [15 oct. 2015].

Zadra, Garbiela Ángela. 2006. “Apuntes sobre el Objeto en la Teoría de J. Lacan”. Psicología vol

5:

14. http://p3.usal.edu.ar/index.php/psico/article/view/1281. [15 oct. 2015]. 